\title{
Intestinal Mucormycosis in Neonates - A Surgical Disease
}

\section{Kargl $\mathbf{S}^{1 *}$, Silye $\mathbf{R}^{2}$ and Pumberger $\mathbf{W}^{1}$}

${ }^{1}$ Department of Pediatric Surgery, Women's and Children's Hospital Linz, Austria ${ }^{2}$ Department of Pathology, Wagner-Jauregg Hospital Linz, Austria

Keywords: Intestinal mucormycosis; Neonates; Abdominal surgery

\section{Introduction}

Mucormycosis is an opportunistic fungal infection typically being associated with diabetes mellitus, leukemia, lymphoma and immunosuppression secondary to transplantation [1]. Mucormycosis may present as intestinal, cutaneous, rhinocerebral, pulmonary and disseminated mucormycosis. Although mucormycosis primarily affects immunocompromised adults, it has also been described in children. Intestinal mucormycosis is found in about one fifth of the affected infants [2].

Mucormycosis is caused by ubiquitous fungus of the family Mucoraceae, including Mucor, Rhizopus and Absidia. The infective forms of these saprophytes are airborne spores which are produced in large amounts. The typical route of entry of these microorganisms into the human body is by inhalation of the infectious spores; ingestion or even percutaneous routes are further possible ways of infection.

In neonates, intestinal mucormycosis is rare and may lead to fatal consequences in most cases. Based on two instructive cases we describe the rare occurrence of intestinal mucormycosis in neonates and discuss the sparse literature on this subject.

\section{Case 1}

A male child was delivered by caesarean section at 36 weeks of gestation because of a prenatally detected complex heart defect. A few days after surgical correction of critical aortic valve stenosis at the age of three weeks the child presented with respiratory distress. Pneumonia was suspected. Sputum cytology revealed Klebsiella pneumoniae and Mucor sp., a fungus deriving from the family of Mucoraceae. A subsequently gained stool culture was positive for Mucor sp. too. Because of the presence of this opportunistic fungus in the respiratory and gastrointestinal tract the seriously ill child was treated with systemic liposomal amphotericin B (2 mg/kg body weight/day) additionally to the antibiotic treatment. After one week of fever and recurring abdominal complaints the child presented with sudden onset of fecal vomiting. In the absence of free air on abdominal X-ray laparotomy was performed and revealed a large abscess in the bursa omentalis. A closed perforation of the stomach had found continuity into the ileum resulting in a gastro-ileal fistula. The gastric perforation was excised and closed with interrupted sutures. A short segment of ileum had to be resected with subsequent end-to-end anastomosis. Because of impossible primary abdominal closure vacuum assisted abdominal closure system (VAC) was used. Histopathology of the specimen revealed invasive mucormycosis. The VAC system was changed every third day and after two weeks we were able to close the abdomen. No major residual lesions were noticed in one year follow up.

\section{Case 2}

Cesarean section was performed at 38 weeks of gestation because of fetal heart rate abnormalities. After severe peripartal asphyxia the boy was stabilized and the child was diagnosed with tetralogy of fallot. Surgical correction of the cardiac defect was performed at two weeks of age. One week later the child presented with distended abdomen and signs of sepsis. Abdominal X-ray showed distended bowel loops but no signs of perforation. Laparotomy was performed because of progressive deterioration and revealed necrotic areas within the terminal ileum and the ascending colon were found. After ileostomy formation and resection of the necrotic bowel segments primary closure of the abdomen was possible. We interpreted our findings as signs of necrotizing enterocolitis but histology demonstrated an invasive fungal disease. Confirmation of invasive mucormycosis was made by histopathological workup of the specimen revealing massive occurrence of invasive hyphes. The tissue was cultured and Rhizopus sp. was identified following micro-morphological and physiological. Intra abdominal fluids were cultured and were also positive for Rhizopus $s p$. Two days later a second look showed dramatic progression of the disease: further segments of ileum and jejunum had turned necrotic and therefore had to be resected. Additionally clay colored necrosis was found in segment VIII of the liver (Figure 1). Because of the extensive intra abdominal damage we decided to use a VAC for abdominal closure (Figure 2). Two days later the child died because of dissemination of invasive fungal disease. Autopsy demonstrated disseminated mucormycosis (Figures 3a-3c) involving gut, liver, spleen and kidneys but pulmonary mucormycosis was not present.

\section{Discussion}

During the last years, especially from the Indian subcontinent several reports of intestinal mucormycosis affecting neonates were noticed [3-7]. It seems, that prematurity is the most important risk factor in neonatal mucormycosis. Preterm infants have an immature immune system and their fragile skin may allow microorganisms to invade. It has been suggested that warmth and high humidity in enclosed incubators could favor fungal infection [8]. Microscopically the fungal filaments present with right-angle branching of the hypes. They tend to invade small vessels thus leading to vasculitis and thrombosis adding additional damage to the affected intestines. Furthermore fungal

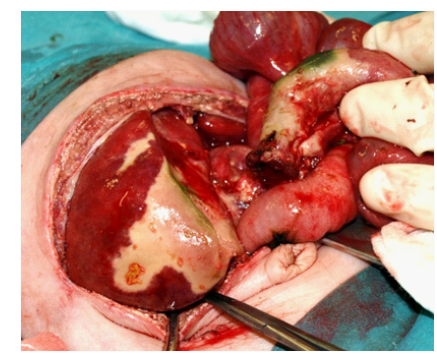

Figure 1: Second look, intraoperative situs: clay-colored lesions in liver and small bowel. Intestinal mucormycosis in Case 2.

*Corresponding author: Simon Kargl, Department of Pediatric Surgery, Women's and Children's Hospital Linz, Krankenhausstrasse 26-30, A-4020 LINZ, Austria, Tel: ++43-50554-63-23305; Fax: ++43-50554-63-26824; E-mail: simon.kargl@gespag.at

Received February 19, 2013; Accepted March 26, 2013; Published April 03, 2013

Citation: Kargl S, Silye R, Pumberger W (2013) Intestinal Mucormycosis in Neonates - A Surgical Disease. Surgery S6: 001. doi:10.4172/2161-1076.S6-001

Copyright: ( $2013 \mathrm{Kargl} \mathrm{S,} \mathrm{et} \mathrm{al.} \mathrm{This} \mathrm{is} \mathrm{an} \mathrm{open-access} \mathrm{article} \mathrm{distributed} \mathrm{under}$ the terms of the Creative Commons Attribution License, which permits unrestricted use, distribution, and reproduction in any medium, provided the original author and source are credited. 


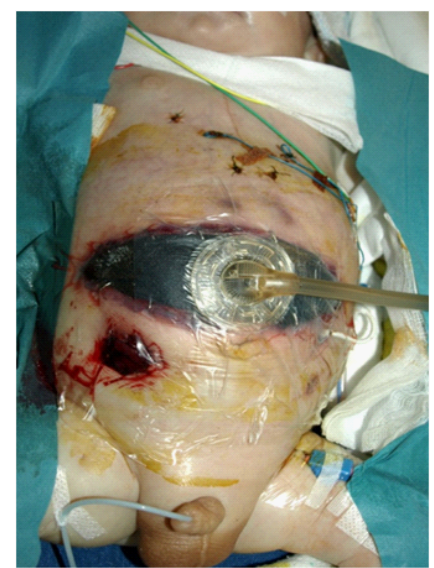

Figure 2: Patient with complex congenital heart defect and intestinal mucormycosis. Stomaformation and vacuum assisted abdominal closure were necessary.

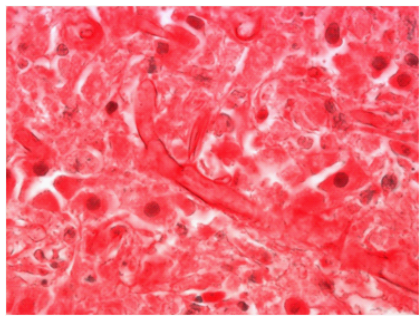

Figure 3a: Liver specimen in a patient with intestinal mucormycosis. Fungal filaments invading hepatic tissue. Right-angle branching of the hypes, liver necrosis. HE100X.

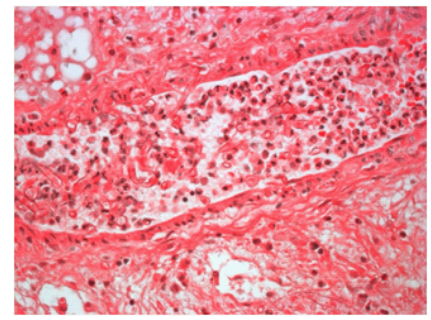

Figure 3b: Intravasal fungal filaments and hypes, HE 40X.

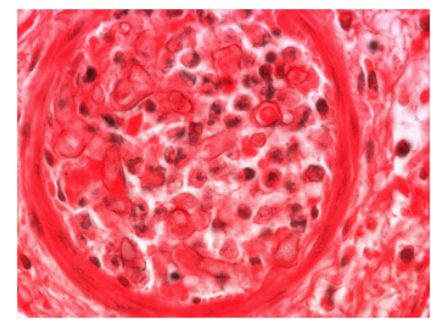

Figure 3c: Intravasalhyphes, HE 100X

angioinvasion promotes hematogenic metastasis causing disseminated disease. Intestinal mucormycosis creates ulcerating and necrotic lesions that might extend rapidly. In neonatal intestinal mucormycosis the colon is the predilection site, whereas in children and adults the stomach is affected in the majority of the cases [9].
Nosocomial infections in neonatal intensive care units are possible: cutaneous infections with Rhizopus have been reported to arise from wooden tongue depressors that were used as splints for venous cannulation sites [10] as well as from contaminated adhesive bandages [11]. Todd et al. described a child infected with Mucor via parenteral nutrition [12].

Our two cases meet the criteria of a nosocomial infectious disease. Both patients were treated in the same unit after open cardiac surgery, although intestinal mucormycosis occurred with a time difference of 12 months. Basically, fungus of the order mucorales are ubiquitous and have been demonstrated in air samples of hospital settings [13].

Although in the majority of cases intestinal mucormycosis is associated with prematurity both our patients were term born babies. Both infants were born with a congenital heart defect. In children with congenital heart defects splanchnic hypoperfusion may occur due to shifting of blood from the intestines to brain, heart and adrenal gland. In our patients transient intestinal ischemia might have increased the susceptibility of the intestine for invasive mucormycosis. Open cardiac bypass surgery was necessary in both infants and might have induced mucosal changes favoring the development of an opportunistic intestinal infection $[14,15]$. Corresponding to these findings Nichol et al. reported a preterm female infant presenting with intestinal mucormycosis five days after being weaned off the extracorporeal membrane oxigenation (ECMO), suggesting a similar pathogenesis [16].

Diagnosis of intestinal mucormycosis in neonates is challenging due to the rather unspecific intestinal complaints. Clinical signs and symptoms might start at the first day of life [17]. Patients present with abdominal distention, (bilious) vomiting, constipation, fever and lethargy. These symptoms are often interpreted as necrotizing enterocolitis [18-22]. In the presence of bilious vomiting the possibility of a volvulus must be taken into account. The frequent involvement of the colon might lead to a suspected diagnosis of toxic megacolon.

The diagnosis of intestinal mucormycosis is generally uncommon prior to surgery. As the oropharynx is the suspected site of entry into the gastrointestinal tract lingual involvement in intestinal mucormycosis is a possible feature not to be missed at clinical examination [23]. In rare cases, fungal hypes may be detected in oropharyngeal smear test, sputum and fecal culture. Positive fecal culture does not prove invasive intestinal mucormycosis and there is a high false-negative rate. Until now, no reliable and clinically useful serological test for mucormycosis exists [13]. A positive culture simply shows the presence of fungus, therefore the diagnosis of invasive mucormycosis has to be made by histopathological examination of the specimen. The specimens have to be processed rapidly and the pathologist must alert clinicians when fungal hypes are found.

The frequent interpretation of neonatal intestinal mucormycosis as necrotizing enters colitis involves significant risks for the patient. Non-operative management of suspected NEC with bowel rest and antibiotics leads to a fatal delay in adequate treatment of these children.

Radiological signs in neonates with intestinal mucormycosis are not characteristic. In the early stage intestinal mucormycosis does not present with intestinal pneumatosis - a characteristic radiological sign of NEC- while in advanced disease bacterial involvement might lead to intestinal pneumatosis [24]. In abdominal ultrasound affected bowel loops present with a thickening of the bowel wall. Free air seen in abdominal plain films indicates intestinal perforation.

Zaotis et al. reported in a review of $2007,100 \%$ mortality for 
children with intestinal zygomycosis (Zygomycetes is the class of the order Mucorales and Entomophtorales). They concluded that surgery did not improve survival [2]. Although a few cases of neonates with gastrointestinal mucormycosis surviving due to aggressive surgery have been reported $[7-9,18,22]$. Treatment of neonatal mucormycosis consists in surgery and systemic antifungal drugs. Amphotericin B is the drug of choice. It is noticeable that systemic amphotericin did not prevent development of severe intestinal mucormycosis in one of our patient (case 1). These findings underline the significance of timely surgical treatment. In our opinion progressive abdominal complaints combined with general signs of deterioration necessitates exploratory laparotomy. In the presence of radiological signs of free intraperitoneal air laparotomy is mandatory. The mainstay in treatment of intestinal mucormycosis lays in adequate surgical debridement to reduce the fungal load and prevent dissemination. In both our cases we used vacuum assisted closure of the abdomen because of extensive intra abdominal inflammatory destructions.

Mortality of intestinal mucormycosis is high and depends on the extent of the disease. Whereas localized mucormycosis provides a possible chance of surgical disease-control, disseminated disease nearly always leads to fatal consequences.

\section{Conclusion}

Intestinal mucormycosis is a rare emergency of the gastrointestinal tract in neonates. Diagnosis remains difficult and mortality is high. Prematurity is an accepted risk factor but lethal mucormycosis might also occur in term born neonates with congenital heart disease. While some authors have defined intestinal mucormycosis as a variant of NEC it is important to say that conservative treatment as in early NEC, consisting in antibiotic treatment and bowel rest, is not sufficient for intestinal mucormycosis. Clinical signs of NEC in children with absent radiological NEC characteristics must raise suspicion for mucormycosis. Antifungal therapy in addition to antibiotic treatment has to be considered early. Aggressive surgical debridement of affected tissue might raise the chance to survive.

\section{References}

1. Petrikkos G, Skiada A, Lortholary O, Roilides E, Walsh TJ, et al. (2012) Epidemiology and clinical manifestations of mucormycosis. Clin Infect Dis 54: S23-34

2. Zaoutis TE, Roilides E, Chiou CC, Buchanan WL, Knudsen TA, et al. (2007) Zygomycosis in children: a systematic review and analysis of reported cases. Pediatr Infect Dis J 26: 723-727.

3. Sarin YK (2010) Intestinal mucormycosis in a neonate: A case report and review. J Indian Assoc Pediatr Surg 15: 98-100.

4. Choudhury M, Kahkashan E, Choudhury SR (2007) Neonatal gastrointestinal mucormycosis--a case report. Trop Gastroenterol 28: 81-82.

5. Budhiraja S, Sood N, Singla S, Gupta V (2007) Gastrointestinal mucormycosis in a neonate: Survival without antifungal therapy. Indian J Gastroenterol 26: 198

6. Agarwal K, Sharma M, Singh S, Jain M (2006) Antemortem diagnosis of gastrointestinal mucormycosis in neonates: report of two cases and review of literature. Indian J Pathol Microbiol 49: 430-432.
7. Patra S, Vij M, Chirla DK, Kumar N, Samal SC (2012) Unsuspected invasive neonatal gastrointestinal mucormycosis: A clinicopathological study of six cases from a tertiary care hospital. J Indian Assoc Pediatr Surg 17: 153-156.

8. Kecskes S, Reynolds G, Bennett G (1997) Survival after gastrointestinal mucormycosis in a neonate. J Paediatr Child Health 33: 356-359.

9. Dhingra KK, Mandal S, Khurana N (2008) Unsuspected intestinal mucormycosis in a neonate presenting as necrotizing enterocolitis (NEC). Eur J Pediatr Surg 18: $119-120$.

10. Mitchell SJ, Gray J, Morgan ME, Hocking MD, Durbin GM (1996) Nosocomial infection with Rhizopus microsporus in preterm infants: association with wooden tongue depressors. Lancet 348: 441-443.

11. Dennis JE, Rhodes KH, Cooney DR, Roberts GD (1980) Nosocomical Rhizopus infection (zygomycosis) in children. J Pediatr 96: 824-828.

12. Todd NJ, Millar MR, Dealler SR, Wilkins S (1990) Inadvertent intravenous infusion of Mucor during parenteral feeding of a neonate. J Hosp Infect 15 295-297.

13. Ribes JA, Vanover-Sams CL, Baker DJ (2000) Zygomycetes in human disease Clin Microbiol Rev 13: 236-301.

14. Booker PD, Romer H, Franks R (1996) Gut mucosal perfusion in neonates undergoing cardiopulmonary bypass. Br J Anaesth 77: 597-602.

15. Malagon I, Onkenhout W, Klok G, van der Poel PF, Bovill JG, et al. (2005) Gut permeability in paediatric cardiac surgery. $\mathrm{Br} \mathrm{J}$ Anaesth 94: 181-185.

16. Nichol PF, Corliss RF, Rajpal S, Helin M, Lund DP (2004) Perforation of the appendix from intestinal mucormycosis in a neonate. J Pediatr Surg 39: 1133 1135.

17. Kataria R, Gupta DK, Mitra DK (1995) Colonic mucormycosis in the neonate Pediatr Surg Int 10: 271-273.

18. Jain D, Kohli K (2009) Neonatal gastrointestinal mucormycosis clinically mimicking necrotizing enterocolitis. Eur J Pediatr Surg 19: 405-407.

19. Ozturk MA, Akin MA, Deniz K, Turan C, Efendioglu B, et al. (2011) Neonatal gastrointestinal mucormycosis in an asphyxiated premature newborn. Turk Pediatr 53: 705-708.

20. Alexander P, Alladi A, Correa M, D'Cruz AJ (2005) Neonatal colonic mucormycosis--a tropical perspective. J Trop Pediatr 51: 54-59.

21. Diven SC, Angel CA, Hawkins HK, Rowen JL, Shattuck KE (2004) Intestina zygomycosis due to Absidia corymbifera mimicking necrotizing enterocolitis in a preterm neonate. J Perinatol 24: 794-796.

22. Siu KL, Lee WH (2004) A rare cause of intestinal perforation in an extreme low birth weight infant--gastrointestinal mucormycosis: a case report. J Perinato 24: 319-321.

23. Gupta R, Parelkar SV, Oak S, Sanghvi B, Prakash A (2011) Neonatal lingua and gastrointestinal mucormycosis in a case of low anorectal malformation-a rare presentation. J Pediatr Surg 46: 745-748.

24. Michalak DM, Cooney DR, Rhodes KH, Telander RL, Kleinberg F (1980) Gastrointestinal mucormycoses in infants and children: a cause of gangrenous intestinal cellulitis and perforation. J Pediatr Surg 15: 320-324.
This article was originally published in a special issue, Neonatal Surgery for Congenital Malformations handled by Editor. Dr. Medoff Cooper- Barbara, The Childrens Hospital of Philadelphia, USA 